\title{
Tribología de recubrimientos Cermet/NiCrBSi depositados mediante HVOF
}

\author{
J.M. GUILEMANY, J. NIN, C. LORENZANA, J. M. MIGUEL, J.R. MIGUEL \\ CPT, Centro de Proyección Térmica. Ingeniería de Materiales. Dept. Enginyeria Química i Metal·lúrgia. Universitat de Barcelona. 08028. Barcelona.
}

\begin{abstract}
El trabajo consta de un completo estudio tribológico del sistema WX que consiste en la mezcla mecánica de WC-12Co y NiCrBSi en diferentes proporciones: $20 \% \mathrm{NiCrBSi}$ (W2), $40 \% \mathrm{NiCrBSi}$ (W4) y $60 \% \mathrm{NiCrBSi}$ (W6). Los recubrimientos se han obtenido por proyección térmica de alta velocidad (HVOF). Para todos los sistemas, el coeficiente de fricción es menor que 0.5 y la energía intercambiada entre el par friccionante y el recubrimiento es inferior a $10 \mathrm{KJ}$. Para cuantificar el desgaste por fricción se han utilizado la profundidad de la huella y el volumen perdido durante el ensayo, obtenidos mediante Interferometría de Barrido de Luz Blanca (SLWI) y SEM. La profundidad de la huella sigue una evolución directamente proporcional al contenido en NiCrBSi. Un mayor contenido en WC-12Co aumenta la resistencia al desgaste por fricción y disminuye la velocidad de desgaste por abrasión (ensayo Rubber Wheel). En los recubrimientos obtenidos, no se observa difusión entre las fases, la adherencia con el sustrato es excelente, la porosidad menor al $2 \%$ y se produce un incremento de la dureza del recubrimiento debido al endurecimiento por trabajo de la matriz causada por impacto.
\end{abstract}

Palabras clave: Proyección térmica HVOF, WC-12Co, NiCrBSi, resistencia al desgaste, conformado.

\section{Tribology of Cermet/NiCrBSi coatings sprayed by HVOF.}

This work consists on a deep tribological study of the WX system composed by a mechanical blend in different compositions of NiCrBSi and WC-12Co powders: $20 \% \mathrm{NiCrBSi}$ (W2), $40 \% \mathrm{NiCrBSi}$ (W4) y $60 \% \mathrm{NiCrBSi}$ (W6). The coatings have been obtained by high velocity oxy-fuel process (HVOF). The measurements made by Ball-On-Disk test are: the friction coefficient is lower than 0.5 and the exchanged energy between the counterparts is under $10 \mathrm{KJ}$. To quantify the friction wear rate, the volume loss and the track depth, Scanning White Light Interferometry and SEM have been used. The track depth is proportional to the amount of NiCrBSi. A higher percentage of WC-12Co increases the friction wear resistance and decreases the abrasion wear rate (Rubber Wheel test). In all the coatings studied, no diffusion processes are found between the mixed phases, the adhesion between the coatings and the substrate is excellent, the porosity level is below $2 \%$ and an increase of microhardness of the coating due to a strengthening of the matrix produced by impacts of solid particles, takes place.

Keywords: Thermal Spray HVOF, WC-12Co, NiCrBSi, wear resistance, forming.

\section{INTRODUCCIÓN}

Los recubrimientos obtenidos por proyección térmica de alta velocidad (HVOF) son utilizados para mejorar la resistencia al desgaste en muchas aplicaciones industriales [1,2]. Un proceso desarrollado en el Centro de Proyección Térmica (CPT) de la Universidad de Barcelona permite obtener formas autosostenidas utilizando materiales resistentes al desgaste mediante la técnica de alta velocidad (HVOF) [3].

Para obtener formas autosostenidas resistentes al desgaste se han utilizado mezclas mecánicas de polvos de WC-12Co con NiCrBSi. Las partículas de CERMET tienen buena resistencia al desgaste abrasivo a elevadas temperaturas [4,5], mientras que la superaleación se añade para mejorar la tenacidad de la pieza y la resistencia al desgaste adhesivo [6]. Ambos materiales poseen elevada resistencia a la corrosión. Todas estas características permiten obtener piezas autosostenidas con una buena combinación de propiedades.

Las propiedades tecnológicas más importantes son la dureza y la resistencia a la corrosión y al desgaste. El estudio de estas propiedades es necesario para comparar los nuevos materiales con los existentes y determinar los campos de aplicación.

\section{EXPERIMENTAL}

Se han proyectado 3 mezclas mecánicas de proporciones 80/20, 60/40, 40/60 en volumen de WC-12Co (Metco 72-FNS) y NiCrBSi (Amdry 4724) respectivamente. La nomenclatura es la siguiente: W2 (20\% NiCrBSi), W4 (40\% NiCrBSi) y W6 (60\% NiCrBSi). La distribu- ción granulométrica de los dos materiales de partida es de $34.6 \mu \mathrm{m}$ de valor medio y el $80 \%$ del volumen analizado entre 14.6 y $56.3 \mu \mathrm{m}$ para el carburo y de $37.4 \mu \mathrm{m}$ de media con el $80 \%$ del total analizado entre 27.3 y $48.9 \mu \mathrm{m}$ para la superaleación. Las piezas y los recubrimientos se han obtenido con un equipo de proyección de alta velocidad Sultzer Metco CDS 100, utilizando propileno como combustible y oxígeno como comburente.

La caracterización microestructural incluye microscopía óptica, electrónica de barrido (SEM), análisis de imagen, análisis de las fases presentes por DRX y EDS (espectroscipía de energía dispersiva) y medidas de microdureza (HVN 300g). Los ensayos de fricción han sido realizados con un Ball-On-Disk (ASTM G99-90) a una distancia de deslizamiento de $1000 \mathrm{~m}$, con un diámetro de giro de $16 \mathrm{~mm}$, una velocidad de deslizamiento de $0.11 \mathrm{~m} \cdot \mathrm{s}^{-1}$ y bajo una carga estática de $15 \mathrm{~N}$. El par friccionante consiste en una bola de metal duro de $11 \mathrm{~mm}$ de diámetro. Para este ensayo la superficie de la probeta ensayada se prepara hasta alcanzar una rugosidad superficial de $0.2 \mu \mathrm{m}$. Los caminos de desgaste se han estudiado utilizando SEM y Interferometría de Luz Blanca (SWLI).

Los ensayos de desgaste abrasivo se han llevado a cabo utilizando un equipo de Rubber Wheel (ASTM G65-91) con una carga de 50N, utilizando corindón de grado 32 a un flujo de $32 \mathrm{~g} \cdot \mathrm{min}^{-1}$. Para determinar la resistencia a la corrosión, se han aplicado los ensayos de voltamperometría cíclica y la medida del potencial de circuito abierto de las muestras con una disolución de $3.4 \%$ de $\mathrm{NaCl}$ como medio agresivo. 


\section{RESULTADOS Y DISCUSIÓN}

\subsection{Caracterización de los recubrimientos}

En la figura I se muestran las imágenes obtenidas mediante SEM de los recubrimientos W2, W4 y W6. Como se puede observar en las imágenes la adhesión entre el recubrimiento y el substrato es excelente y la poca porosidad existente es debida a los arranques producidos durante su preparación metalográfica. La porosidad en todos los casos es menor al 2\% (tabla I) pero este valor es relativo ya que contiene la porosidad de la preparación metalográfica que no tiene que ser igual para cada sistema. Para el caso de W4 puede ser que la contribución de la porosidad de preparación en la porosidad total sea mayor que en los otros dos casos dando una porosidad mayor de la esperada.

TABla I. Porosidad DE LOS SISTEMAS MEDIANTE ANÁLISIS DE IMAGEN.

\begin{tabular}{|l|l|l|l|}
\hline Sistema & W2 & W4 & W6 \\
\hline Porosidad $(\%)$ & $1.47 \pm 0.2$ & $1.81 \pm 0.2$ & $1.59 \pm 0.1$ \\
\hline
\end{tabular}

En la imagen (d) de la figura I se puede observar la ausencia de difusión entre los componentes de la microestructura. Los perfiles de concentración de elementos químicos obtenidos por EDS y espectroscopía Auger confirman la ausencia de zona difusiva entre las fases ya que entre las distintas fases existentes hay un cambio brusco de concentración. No se establece ningún tipo de interacción o reacción entre las fases durante el proceso de proyección y por tanto el anclaje entre las dos fases es puramente mecánico [7]. Los difractogramas de los recubrimientos obtenidos muestran que no hay diferencias entre los sistemas WX aunque han aparecido nuevas fases durante el proceso de proyección. En la figura II se pueden ver los difractogramas para las condiciones W4 y W6. La fase de WC presenta picos estrechos y definidos. Es la señal de intensidad 100 del difractograma. Se ha detectado la fase $\mathrm{W}_{2} \mathrm{C}$, también observada por SEM (figura IIIa), que no estaba presente en el material de partida y por lo tanto se ha formado durante el proceso de proyección. Las fases de Co y de Ni presentan picos de poca intensidad e imposibles de diferenciar ente sí. La zona central amorfa o nanocristalina del difractograma, (ángulos $2 \theta$ entre $40^{\circ}$ y $55^{\circ}$ ) proviene de la matriz de NiCrBSi y aumenta de volumen al aumentar la proporción de NiCrBSi de la mezcla. En el caso del NiCrBSi se han detectado precipitados de $\mathrm{CrB}$, también en las imágenes de SEM (figura IIIb) que ya estaban presentes en el polvo de partida. El NiCrBSi no experimenta reacción alguna durante el proceso de proyección.

\subsection{Propiedades Tecnológicas}

\subsubsection{MICRODUREZA}

La tabla II resume los valores de microdureza Vickers a 300 gramos de carga de los sistemas WX, del NiCrBSi y del WC-12Co.
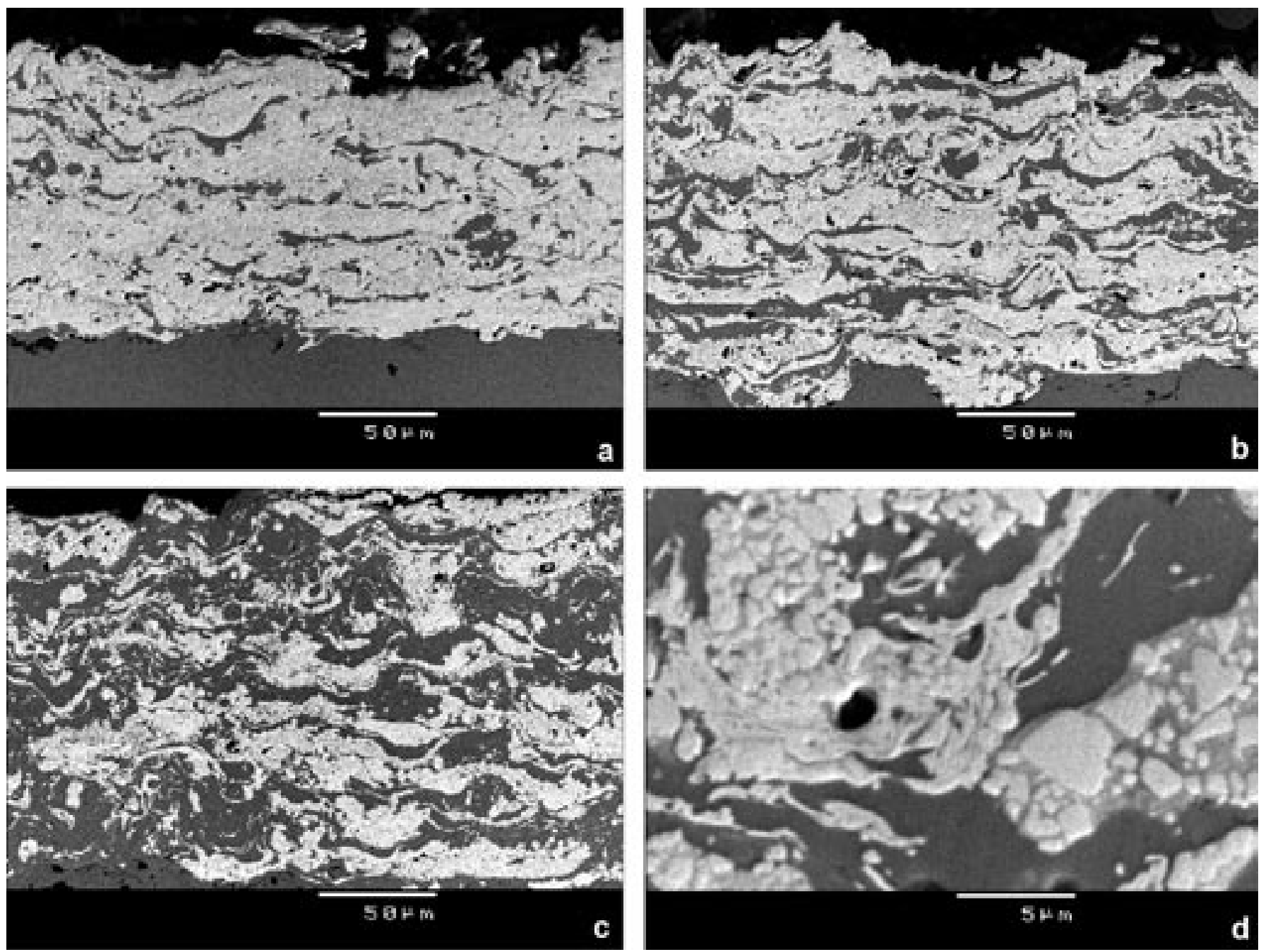

Figura 1. Imágenes de SEM de los recubrimientos obtenidos: W2(a), W4(b), W6(c) e imagen a mayores aumentos de W4(d). 


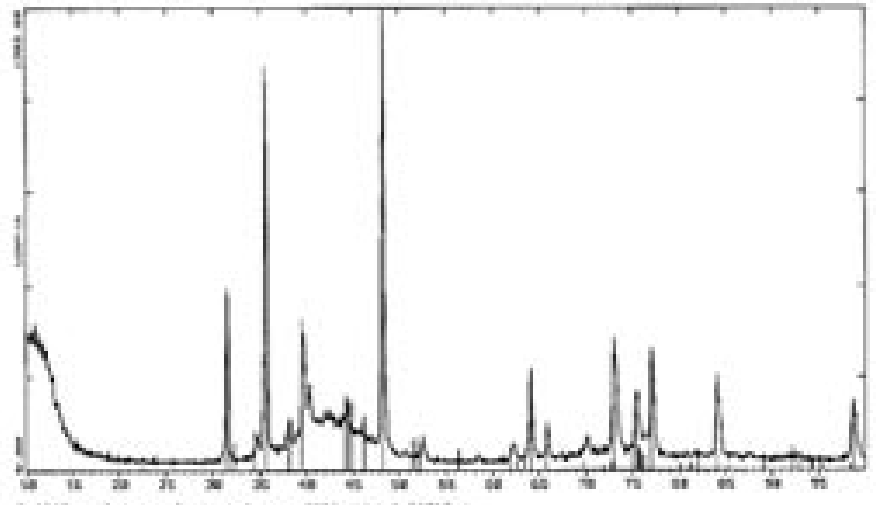

Hen

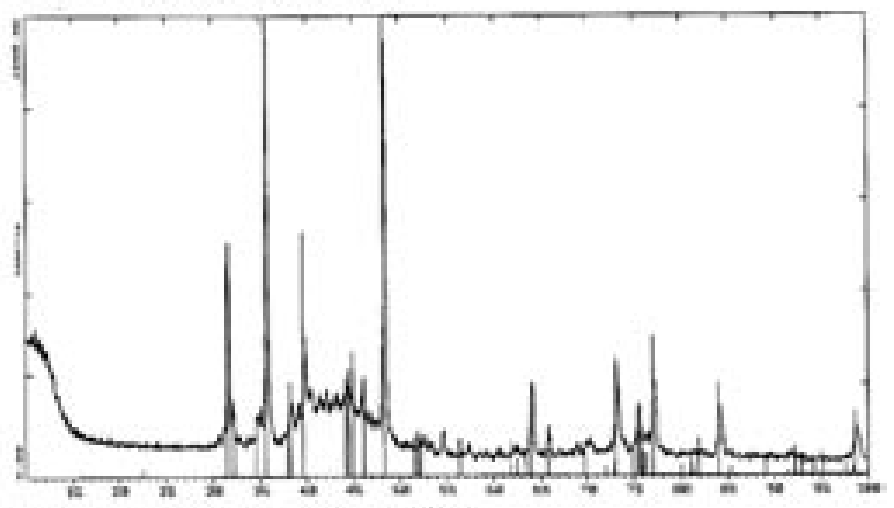

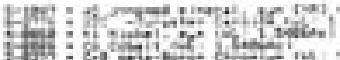

Figura 2. Espectros de DRX para los recubrimientos W4 (arriba) y W6 (abajo).

Se puede observar una mejora de la microdureza en todas las mezclas respecto a los materiales iniciales. La mejora está entre un $15 \%$ (W6) y un 37\% (W4). El incremento de dureza se debe al efecto de endurecimiento de la matriz metálica por el impacto de partículas sólidas [8]. En este caso el impacto de la fase que llega al substrato sin fundir (WC-12Co) crea tensiones sobre la fase metálica ya depositada (NiCrBSi) endureciéndola por deformación plástica. Las partículas de NiCrBSi llegan en estado líquido o pastoso y la deformación, y por lo tanto el endurecimiento que éstas crearan sobre la fase metálica, será menor.

TABLA II. VALORES DE MICRODUREZA VICKERS A 300 GRAMOS.

\begin{tabular}{|l|l|l|l|l|l|}
\hline Sistema & W2 & W4 & W6 & WC-12Co & NiCrBSi \\
\hline HVN $_{300}$ & $1178 \pm 24$ & $1236 \pm 26$ & $1035 \pm 20$ & $898 \pm 27$ & $905 \pm 9$ \\
\hline
\end{tabular}

El recubrimiento con mayor dureza es el W4.Teóricamente el W2 debería ser el más duro porque tiene mayor porcentaje de WC-12Co pero en este caso el porcentaje de fase metálica endurecible (NiCrBSi) es solo del $20 \%$. El sistema W4 es la que mayor dureza presenta. Para este caso la combinación de un $60 \%$ de partículas sólidas que impactan con un $40 \%$ de matriz metálica hacen posible el mayor endurecimiento por impacto. Para el caso del sistema W6, la microdureza es la menor de los tres sistemas debido a que sólo hay un $40 \%$ de partículas sólidas impactando sobre el $60 \%$ de la fase metálica. En W6, habría más impactos de partículas de NiCrBSi sobre la matriz metálica que no impactos de partículas de WC-12Co.
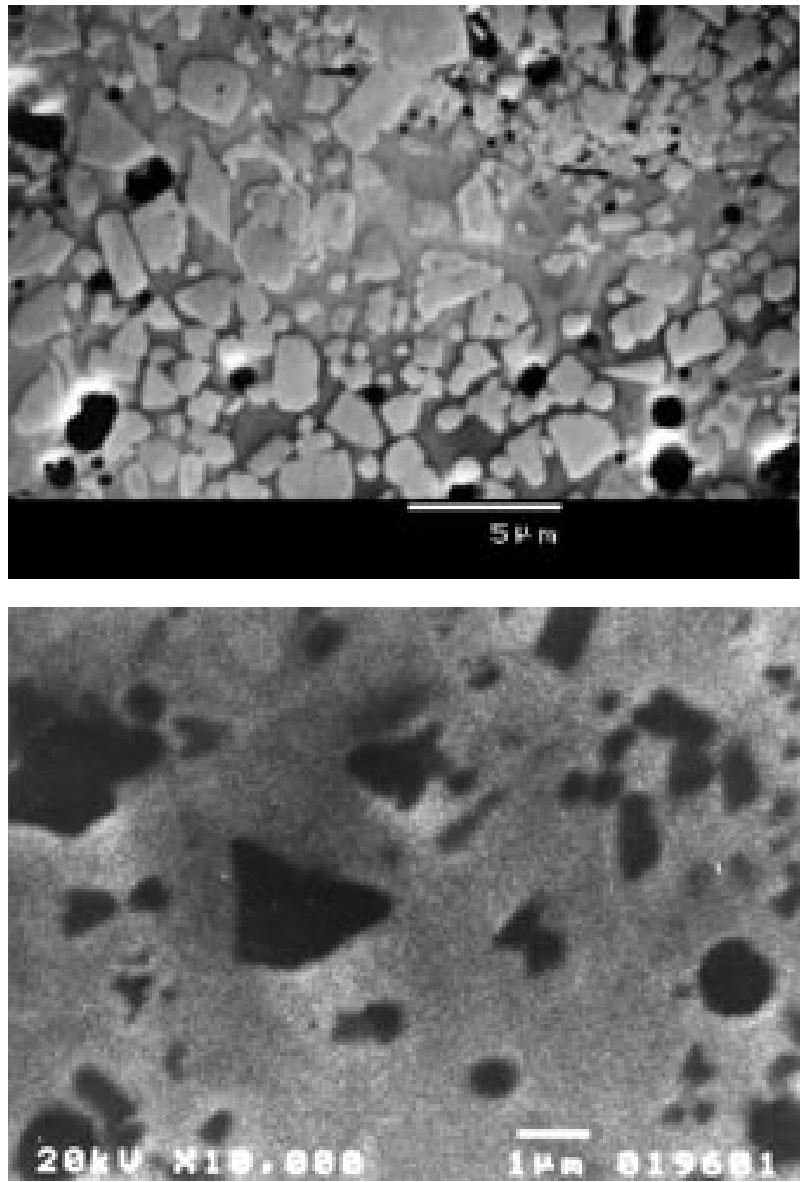

Figura 3. (a): $\mathrm{W}_{2} \mathrm{C}$ en contraste claro alrededor de las partículas poliédricas de WC. (b): Precipitado de $\mathrm{CrB}$ en contraste oscuro disperso en una matriz de $\mathrm{NiCr}$

\subsubsection{Desgaste abrasivo}

La figura IVa muestra los valores de velocidad de desgaste abrasivo obtenidos mediante el ensayo de Rubber Weel. La velocidad de desgaste se expresa con $\mathrm{mm}^{3}$ de recubrimiento perdido por carga aplicada, metro recorrido y minuto de ensayo. En los sistemas WX, un aumento en la proporción de WC-12Co significa una disminución de la velocidad de desgaste. La resistencia al desgaste abrasivo de los sistemas WX es siempre menor que la resistencia de los recubrimientos de WC-12Co aunque para el sistema W2 es muy similar. La resistencia al desgaste abrasivo del NiCrBSi es tan pequeña que se alcanza el acero substrato antes de que se finalice el ensayo.

\subsubsection{Desgaste por fricción}

La figura IVb muestra los valores de los parámetros de desgaste obtenidos con el ensayo Ball-On-Disk. Los datos se han obtenido mediante la utilización de SWLI (figura $\mathbf{V}$ ). El volumen perdido y la profundidad de huella para el NiCrBSi $\left(1.08 \mathrm{~mm}^{3}\right.$ y $25,7 \mu \mathrm{m}$ respectivamente) son ordenes de magnitud mayores que los de los demás sistemas y no se muestran en la figura IVb.

El recubrimiento con mayor resistencia al desgaste por fricción es el W2. Los sistemas W4 y W6 muestran resistencias a la fricción similares entre si. No se aprecia perdida de volumen para el caso del WC-12Co. El WC-12Co tiene una elevada resistencia al desgaste por 


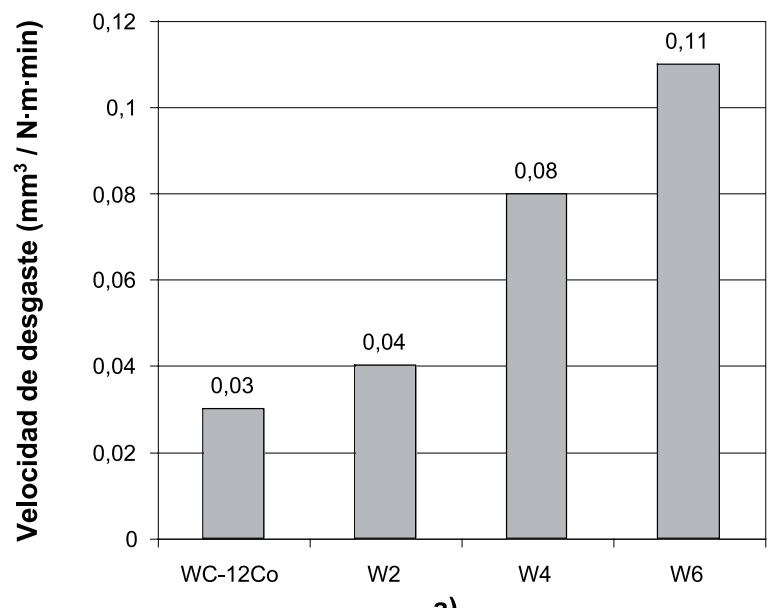

a)

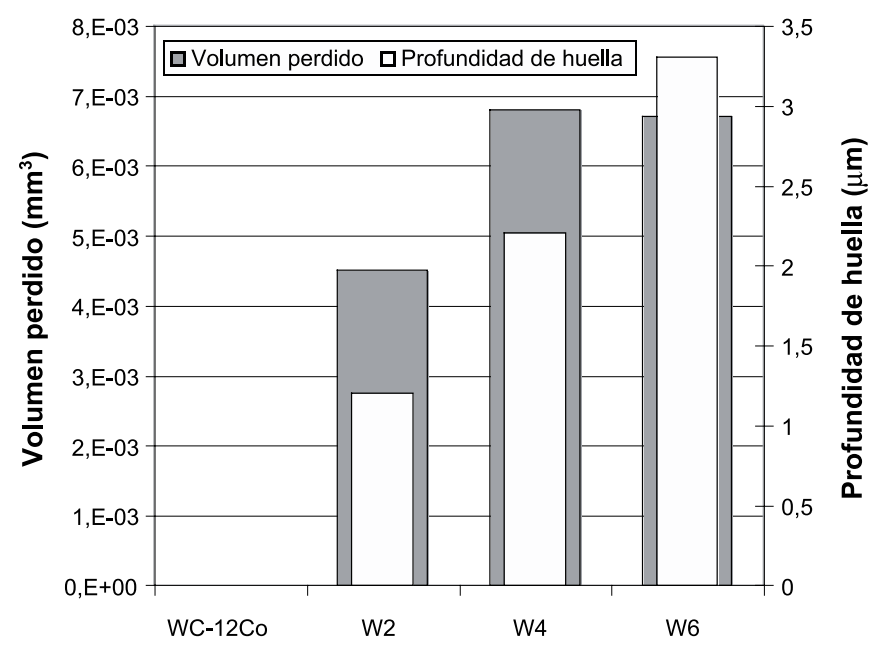

b)

Figura 4. (a) Velocidad de desgaste abrasivo mediante ensayo Rubber Wheel. (b) Volumen perdido y profundidad de huella mediante ensayo Ball-On-Disk.

fricción por la formación durante el ensayo de fases lubricantes como la $\mathrm{WO}_{3}$ [9]. Un mayor contenido en WC-12Co en los sistemas aumenta por lo tanto la resistencia a este tipo de fricción.

La profundidad de la huella aumenta con el volumen de NiCrBSi.

La tabla III muestra los coeficientes de fricción $(\mu)$ a 200 metros $\left(\mu_{200}\right)$ obtenidos mediante ensayo Ball-On-Disk de todos los sistemas WX y de los dos materiales de partida sin mezclar. Para todos los casos el coeficiente está por debajo de 0.5 y la energía intercambiada por debajo de $10 \mathrm{KJ}$. El coeficiente de fricción para los sistemas WX aumenta con el contenido de NiCrBSi al pasar del 20 al $40 \%$ y se mantiene constante para el caso del $60 \%$ de fase metálica. El WC-12Co tiene el coeficiente menor de todos mientras que el NiCrBSi tiene el coeficiente de fricción mayor. Este hecho se explica por la relación existente entre el volumen perdido (profundidad y anchura del camino de desgaste) y el coeficiente de fricción. Cuanto más penetra la bola friccionante en el recubrimiento, mayor superficie de contacto existe entre ambos. A mayor superficie de contacto mayor la temperatura generada en la interfase y mayor es el desgaste [10]. La evolución del coeficiente de fricción y el volumen perdido y la profundidad es paralela. Los sistemas W4 y W6 presentan coeficientes parecidos y presentan también volúmenes perdidos similares.

TABLA III. CoEfiCientes de FRICCIÓN $\left(\mu_{200}\right)$ A 200 METROS DE ENSAYO Y ENERGÍA INTERCAMBIADA.

\begin{tabular}{|l|l|l|l|l|l|}
\hline Sistema & W2 & W4 & W6 & WC-12Co & NiCrBSi \\
\hline$\mu_{200}$ & 0.33 & 0.40 & 0.40 & 0.32 & 0.46 \\
\hline Energía (KJ) & 4.83 & 6.40 & 6.74 & 4.50 & 6.94 \\
\hline
\end{tabular}

\subsection{Resistencia a la corrosión}

La figura VI muestra el resultado de los ensayos electroquímicos para los sistemas WX. Los valores de potencial de circuito abierto $\left(\mathrm{E}_{\mathrm{OC}}\right)$ para los WX son: $-439 \mathrm{mV}$ para $\mathrm{W} 2,-436 \mathrm{mV}$ para $\mathrm{W} 4 \mathrm{y}-416 \mathrm{mV}$ para W6. Dichos valores están por encima del $\mathrm{E}_{\mathrm{OC}}$ del substrato y muestran estabilidad durante las primeras 5 horas de inmersión. Los resultados de la voltamperometría cíclica $(\mathrm{Cv})$ indican la rotura de la pasividad de los sistemas entre 300 y $400 \mathrm{mV}$. No obstante se observa una intensidad de corrosión menor para el sistema W6 durante el periodo de pasividad debido a que el sistema W6 tiene mayor proporción de fase metálica NiCrBSi.

Los procesos de corrosión se localizan preferentemente en las partículas de WC-12Co [11], y concretamente en la matriz de Co que envuelve a las partículas de WC y por tanto a menor volumen de fase WC-12Co mayor será la resistencia a la corrosión.

\section{CONCLUSIONES}

- La adherencia entre sustrato y recubrimiento es excelente. El anclaje es puramente mecánico ya que no se observa por SEM o por EDS difusión entre las fases presentes o entre el recubrimiento y el sustrato.

- En todos los casos la microestructura es compacta con un nivel de porosidad inferior la $2 \%$.

- La fase $\mathrm{W}_{2} \mathrm{C}$ se crea durante el proceso de proyección. El NiCrBSi no sufre ninguna alteración composicional durante la proyección. La fase $\mathrm{CrB}$ se ha detectado en el material de inicio.

- En todos los sistemas WX, se produce un aumento de la microdureza entre el 15 y el $37 \%$ respecto a los materiales de inicio debido a
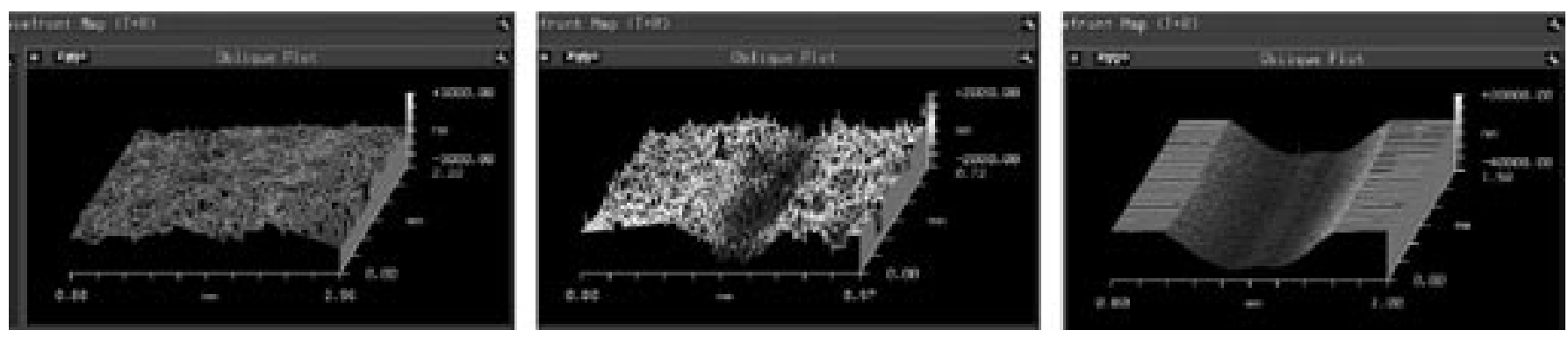

Figura 5. Imágenes de los caminos de desgaste mediante SWLI de los recubrimientos: WC-12Co (izquierda), W2 (centro) y NiCrBSi (derecha). 

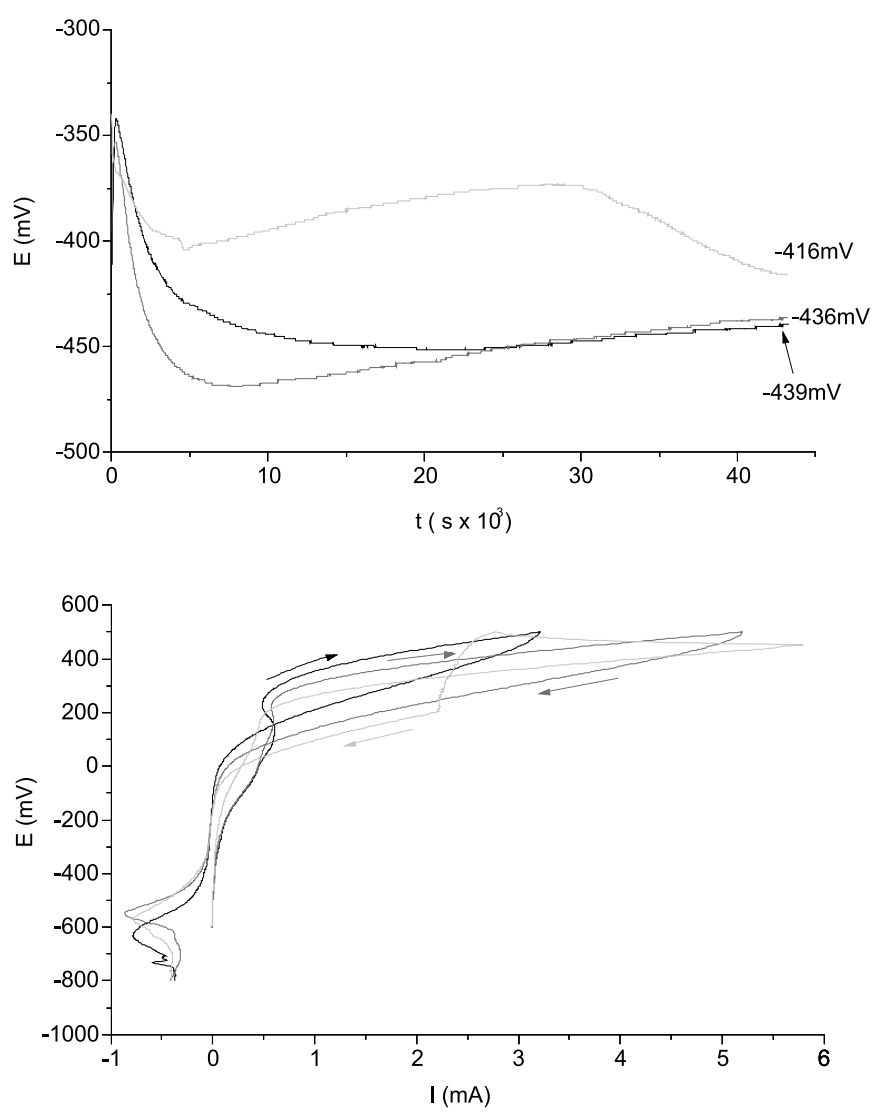

Figura 6. Ensayos electroquímicos. Circuito abierto (arriba) y voltamperometría cíclica (abajo). Leyenda: W2 en negro, W4 en rojo y W6 en verde.

procesos de endurecimiento de la matriz por impacto.

- En los sistemas WX, un aumento en la proporción de WC-12Co significa un aumento a la resistencia al desgaste abrasivo.

- Un mayor contenido en WC-12Co en los sistemas aumenta la resistencia al desgaste por fricción. La profundidad de la huella aumenta con el volumen de NiCrBSi así como el coeficiente de fricción, sobre todo al pasar de un $20 \%$ de $\mathrm{NiCrBSi}$.

- El sistema W6 es el que presenta mayor resistencia a la corrosión ya que el volumen de WC-12Co es menor. El ataque se localiza en la matriz de Co alrededor de las partículas de WC-12Co.

\section{AGRADECIMIENTOS}

J. Nin agradece a la Universitat de Barcelona por la concesión de la Beca de Recerca i Docencia.

Los autores agradecen a la Generalitat de Catalunya la beca 2000TDOC0048 y el proyecto 2001SGR00145 y al Ministerio de Ciencia y Tecnología por el proyecto MAT2001-3498.

\section{BIBLIOGRAFÍA}

1. Bloggs, J. and A.N. Other: Literature example for manuscripts in DVS volumes of reports. Welding \& Cutting 46, Issue 13, pp. 1156/57. (1999).

2. Pawlowski, L.. The Science and Engineering of Thermal Spray Coatings, Ed. John Wiley \& Sons, ISBN 047195253 2, (1995).

3. Guilemany, J.M. Miguel, J.R. Dougan, M.J. de Paco, J.M. Dong, Z. and Llibre, J. European Patent 21333077, (2000).

4. Guilemany, J.M. and Calero, J.A. “ Estudio del comportamiento mecánico de resistencia al desgaste de recubrimientos de $\mathrm{Cr}_{3} \mathrm{C}_{2}-\mathrm{NiCr}$ obtenidos por HVOF". V Congreso Nacional de Propiedades Mecánicas de Sólidos.Vol 1. ISBN 84- 89349-59-2.. CPDA,S.A.L. Barcelona. Pp. 236-241. (1996).

5. Sonoya, K. Ji, G. and Wang, Y. "Effect of powder type on the relationship between spray parameters and properties of $\mathrm{HVOF}$ sprayed $\mathrm{Cr}_{3} \mathrm{C}_{2}-\mathrm{NiCr}$ Coatings". Proceedings of the $15^{\text {th }}$ International Thermal Spray Conference. ISBN: 0-87170-659-8, Nice, France. Pp. 287/292. (1998).

6. Jia, Y. and Yu, F." A Comparison of properties of NiCrBSi-WC coatings deposited by HVOF and flame spray processes". Proceedings of International Thermal Spray Conference, Kobe, Japan. Pp. 827-832. ISSN 1241-3074. (1995).

7. Guilemany, J.M. Franch, R. Lorenzana, C.1998 Powder Metallurgy World Congress. Granada.Vol4. ISBN 189907209 8. UK. (1998).

8. Tiwari, R. "Thermal spray forming of metals and their composites". Dissertation Abstracts International 53 (6). ISSN 0419-4217. Pp 251. (1992)

9. Fervel, V., Normand, B., Liao, H., Coddet, C. “Friction and wear mechanism of thermally sprayed ceramic and cermet coatings". Surface and Coatings Technology. 111, pp 255-262. (1999).

10. Hutchings I.M, "Tribolgy: friction and wear of engineering materials", Edward Arnold, London, 1992, ISBN: 0-340-56184-x.

11. Guilemany, J.M., de Paco, J., Miguel, J.R, Llibre, J. “Obtention and development of blends of powder to obtain free-standing components produced by HVOF spray forming". Proceedings of the International Thermal Spray Conference'98. ISBN 0-87170-659-8. Nice. France.(1998)

Recibido: 1.2 .03

Aceptado: 30.11 .03 